\title{
Review of: "How pharmacy and medicine students experience the power differential between professions: "Even if the pharmacist knows better, the doctor's decision goes""
}

Ruyi Tong ${ }^{1}$

1 Curtin University of Technology

Potential competing interests: The author(s) declared that no potential competing interests exist.

This article addresses an important, yet under-researched area in the interprofessional field: power and identity. In addition to professional identity, there is a growing literature on interprofessional identity. Nuanced differences exist between interprofessional identity and professional identity. Exploring the interactions among power, (inter)professional identities and interprofessional education may lead to new insights into how discussions about power and identity can be meaningfully integrated into extant interprofessional education programmes and/or into existing pharmacy and medical curricula. 